\title{
-NOTES-
}

\section{QUASI-STATIONARY AIRFOIL THEORY IN SUBSONIC COMPRESSIBLE FLOW*}

\author{
By JOHN W. MILES (University of California, Los Angeles)
}

Summary. A solution of the integral equation for an oscillating, two-dimensional, thin airfoil in a compressible flow (subsonic and inviscid) is obtained by retaining only first order terms in frequency. The results are applied to the calculation of the damping derivative of a tail in rotary motion about a forward center, and it is shown that the damping is considerably less than that calculated on the basis of stationary airfoil theory. A brief investigation of induction effects shows this reduction to be considerably less for a wing of finite aspect ratio.

1. Introduction. The problem to be considered in this paper is the determination of the pressure distribution on a two-dimensional thin airfoil in a compressible flow for the case where the prescribed downwash distribution exhibits a low frequency, harmonic time dependence. (By "low frequency" we imply that only terms of first order in the reduced frequency parameter, $k$, will be retained.) The situation of primary interest is subsonic, compressible flow, since the incompressible ${ }^{1}$ and supersonic ${ }^{2}$ cases have been considered previously.

In studying the disturbed motion of an airplane about its equilibrium position, the aerodynamic forces generally have been calculated on the basis of steady flow. The argument advanced is essentially that the motions are "slow" (i.e., low frequency), so that aerodynamic phase shifts may be neglected. The implicit assumption is that only first order terms in frequency need by retained and that these terms occur only in the (airfoil) displacement. Unfortunately, first order terms also occur in the hydrodynamical equations and will be important, insofar as zero order terms occur in the prescribed downwash.

Also, it must be expected that compressibility effects will be rather more important in non-stationary flows, since the effect of a finite velocity of propagation on the disturbances introduced by the airfoil is to emphasize phase shifts, as represented by both first and second time derivatives in the potential equation. Thus, the linearized (subsonic) compressibility correction of the quasi-stationary results for incompressible flow over a thin airfoil is not effected by simply introducing the Prandtl-Glauert factor $\left(1-M^{2}\right)^{-1 / 2}$. The variation with Mach number is particularly important at supersonic speeds, where the first order (in frequency) terms neglected by steady flow theory may cause a one degree of freedom system to pass from an unstable to a stable state (or conversely) at the transition of some critical value of $M$.

The analysis of quasi-stationary, compressible flow is quite naturally a limiting case of the oscillating airfoil theory first considered by Possio. ${ }^{3}$ Fortunately, a closed form

\footnotetext{
*Received July 18, 1949. The results in this paper were originally announced in the Readers' Forum, J. Aero. Sci. 16, 511 (1949), and the present material is based on work done at the Aerophysics Laboratory, North American Aviation, Inc.

1J. W. Miles, Quasi-stationary thin airfoil theory, J. Aero. Sci. 16, 440 (1949).

${ }^{2}$ J. W. Miles, On harmonic motion at supersonic speeds, J. Aero. Sci. 16, 378-379 (1949).

${ }^{3} \mathrm{C}$. Possio, L'Azione aerodinamica sul profilo oscillante in un fluido compressible a velocita iposonora, L'Aerotechnica, 18, 441-458 (1938).
} 
solution may be given for this limiting case, whereas this has not yet been possible for the more general situation.

2. The Possio integral equation. A thin airfoil is located near $z=0,|x|<1$ in a subsonic, compressible flow of velocity $U$ directed along the positive $x$ axis, as shown in Fig. 1. If $w(x)$ exp $(i \omega t)$ is the prescribed downwash at a point $x$ on this airfoil, $\gamma(x)$

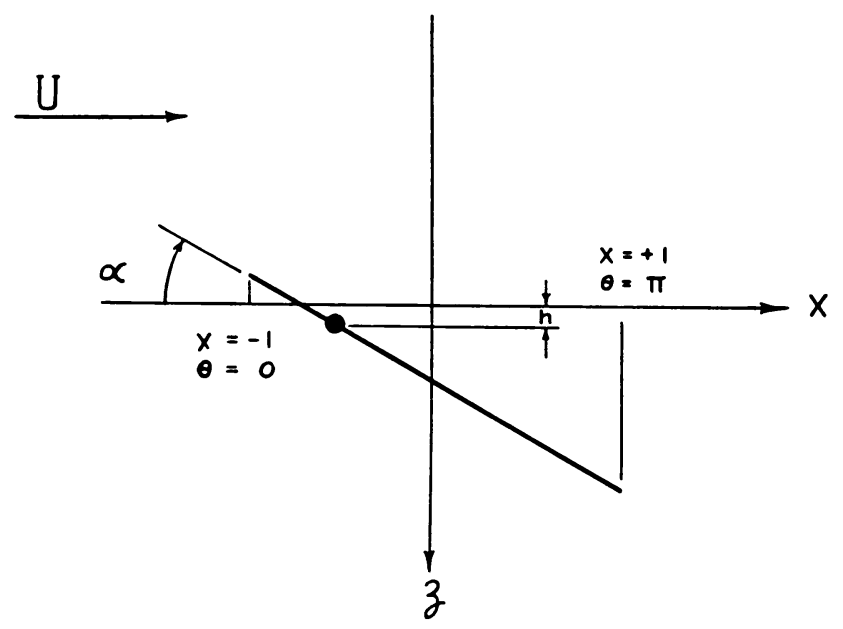

FIg. 1. Thin Airfoil in two-dimensional flow.

$\exp (i \omega t)$ represents the local vorticity associated with this downwash distribution, $M$ is the Mach number, and $k$ is the reduced frequency, the required integral equation between $w$ and $\gamma$ may be written ${ }^{4}$

$$
\begin{gathered}
w(x)=(2 \pi)^{-1}\left(1-M^{2}\right)^{1 / 2} \int_{-1}^{1} d \xi(x-\xi)^{-1} \exp [-i k(x-\xi)] \\
\cdot R\left[M, k\left(1-M^{2}\right)^{-1}(x-\xi)\right] \gamma(\xi) \\
\gamma(1)=0 \\
k=(\omega b / U) \\
R(M, y)=\frac{1}{2} i \pi M y \int_{-\infty}^{y} d u|u|^{-1} \exp (i u) H_{1}^{(2)}(M|u|)
\end{gathered}
$$

where $H_{1}^{(2)}$ is a Hankel function in Watson's notation. ${ }^{5}$ Eq. (2.1b) is the Kutta condition (continuous pressure at the trailing edge) and is required in order to render the solution unique. The semi-chord, $b$, is the unit of length, as indicated in Eq. (2.2)

A discussion of Possio's integral equation and the various methods which have been proposed for its solution, together with a discussion of the analogous supersonic problem, 
has recently been given by Biot and others. ${ }^{6}$ The procedure to be used below is essentially that previously proposed for the case of small Mach number. ${ }^{7}$ In carrying out this solution we find it expedient to introduce the modified downwash and vorticity functions

$$
\begin{aligned}
\mathbf{w}(x) & =\left(1-M^{2}\right)^{-1 / 2} \exp \left(-i M^{2} \mathbf{k} x\right) w(x) \\
\boldsymbol{\gamma}(x) & =\exp \left(-i M^{2} \mathbf{k} x\right) \gamma(x) \\
\mathbf{k} & =\left(1-M^{2}\right)^{-1} k
\end{aligned}
$$

whence Eq. (2.1) transforms to

$$
\begin{gathered}
\mathbf{w}(x)=(2 \pi)^{-1} \int_{-1}^{1} d \xi(x-\xi)^{-1} \exp [-\imath \mathbf{k}(x-\xi)] R[M, \mathbf{k}(x-\xi)] \boldsymbol{\gamma}(\xi) \\
\gamma(1)=0
\end{gathered}
$$

3. Approximate solution to Possio equation. We now set ourselves the problem of solving Eq. (2.7) after neglecting terms of order $\mathbf{k}^{2}$ or higher. The first step in this solution is naturally the expansion of $R(M, y)$ in powers of $y$, with only terms of order $y$ (by order $y^{n}$, we also imply terms of order $y^{n} \ln y$ ) to be retained.

As it stands, $R(M, y)$ is improper for positive values of $y$ due to the singularity of order $u^{-2}$ in the integrand. Actually this difficulty is synthetic and may be removed by integration by parts to obtain a proper integral. ${ }^{8}$ Thus, integrating Eq. (2.3) twice by parts, evaluating the infinite integral with the aid of the Weber-Shafheitlin integrals given by Watson, ${ }^{9}$ multiplying the result through by exp $(-i y)$, and expanding in powers of $y$, we may approximate Eq. (2.7) in the form

$$
\begin{gathered}
\mathbf{w}(x)=(2 \pi)^{-1} \int_{-1}^{1} d \xi\left[(x-\xi)^{-1}+\mathbf{k} h(x-\xi)\right] \boldsymbol{\gamma}(\xi)+O\left(\mathbf{k}^{2}\right) \\
\gamma(1)=0 \\
h(x)=\frac{1}{2} \pi-i[\gamma+\ln (\mathbf{k}|x|)+f(M)] \\
f(M)=\left[1-\left(1-M^{2}\right)^{1 / 2}\right] \ln M+\left(1-M^{2}\right)^{1 / 2} \ln \left[1+\left(1-M^{2}\right)^{1 / 2}\right]-\ln 2
\end{gathered}
$$

where $\gamma$ (without functional dependence, to distinguish from $\gamma(x)$ ) is Euler's constant.

Eq. (3.1) can be solved by iteration, for if $\gamma(x)$ is written $\gamma_{0}(x)+\mathbf{k} \gamma_{1}(x)$ and powers of $k$ equated, the results may be identified as the well known integral equation for thin

${ }^{6}$ S. N. Karp, S. S. Shu, H. Weil, M. A. Biot, Aerodynamics of the oscillating airfoil in compressible flow, F-TR-1167-ND, HQ, AMC, Wright Field, Dayton, Ohio (1947).

${ }^{7} \mathrm{~J}$. W. Miles, $A$ note on a solution to Possio's integral equation for an oscillating airfoil in subsonic flow, Q. Appl. Ma. 7, 213-217 (1949).

${ }^{8} \mathrm{We}$ do not attempt complete rigor here, but we remark that this difficulty is common to many problems of mathematical physics which are formulated as Fredholm integral equations. It usually may be removed by a more thorough derivation, including, in particular, the linearization of the boundary conditions and the associated passage to the limit after appropriate integrations by parts (rather than before, as is implicit in the present case).

loc. cit. 5 , p. 405 , Eqs. (4) and (5). 
airfoils in steady, incompressible flow. While this would be the most direct approach, it may be observed that our problem may be reduced to an equivalent incompressible problem (neglecting terms of order $\mathbf{k}^{2}$ ) if $k$ and Euler's constant $(\gamma)$ are replaced by $\mathbf{k}$ and $\gamma+f(M)$, respectively. Expanding Schwarz's solution for the incompressible problem, ${ }^{10}$ retaining only terms of order $\mathbf{k}$, and substituting $\mathbf{k}, \mathbf{w}$, and $\gamma$, from Eqs. (2.4)-(2.6), in the resulting solution to Eq. (3.1), we obtain the desired approximate solution to Eq. (2.1) in the form

$$
\begin{gathered}
\gamma(-\cos \theta)=\pi^{-1}\left(1-M^{2}\right)^{-1 / 2} \int_{0}^{\pi} d \varphi g(\theta, \varphi) w(-\cos \varphi) \\
g(\theta, \varphi)=2\left[\cot (\theta / 2)+(\cos \theta-\cos \varphi)^{-1} \sin \theta\right] \\
+i \mathbf{k}\left\{\sin \varphi \ln \left[\frac{1-\cos (\theta+\varphi)}{1-\cos (\theta-\varphi)}\right]\right. \\
+2\left[\gamma+\frac{1}{2} i \pi+\ln \left(\frac{1}{2} \mathbf{k}\right)+f(M)-M^{2}\right] \\
\left.\cdot(1-\cos \varphi) \cot \left(\frac{1}{2} \theta\right)\right\}+O\left(\mathbf{k}^{2}\right)
\end{gathered}
$$

4. Lift and moment coefficients. The pressure coefficient on the wing is given by

$$
c_{p}(x)=\left(\frac{1}{2} \rho U^{2}\right)^{-1}[p(x, 0-)-p(x, 0+)]=2 U^{-1} \gamma(x)
$$

The lift and (quarter chord) moment coefficients, referred to the chord and the square of the chord, respectively, are given by

$$
\begin{aligned}
& c_{l}=U^{-1} \int_{0}^{\pi} d \theta \gamma(-\cos \theta) \sin \theta \\
& c_{m}=(2 U)^{-1} \int_{0}^{\pi} d \theta \gamma(-\cos \theta) \sin \theta \cos \theta-\frac{1}{4} c_{l}
\end{aligned}
$$

The downwash due to a displacement $z(x)$, positive down, is given by

$$
w(x)=\frac{D}{D t} z(x)=U\left[z^{\prime}(x)+i k z(x)\right]
$$

Combining Eqs. (3.4), (3.5), and (4.4), substituting in Eqs. (4.2) and (4.3), separating the real and imaginary parts, and introducing $\mathbf{k}$ from Eq. (2.6), we obtain

$$
\begin{gathered}
R l\left(c_{l}\right)=2\left(1-M^{2}\right)^{-1 / 2} \int_{0}^{\pi} d \varphi(1-\cos \varphi) z^{\prime}(-\cos \varphi)+O(k) \\
\operatorname{Im}\left(c_{l}\right)=2 k\left(1-M^{2}\right)^{-1 / 2} \int_{0}^{\pi} d \varphi(1-\cos \varphi) z(-\cos \varphi) \\
\quad+2 k\left(1-M^{2}\right)^{-3 / 2} \int_{0}^{\pi} d \varphi\left\{\sin ^{2} \varphi\right.
\end{gathered}
$$

${ }^{10} \mathrm{~L}$. Schwarz, Berechnung der Druckverteilung einer harmonisch sich verformenden Tragflache in ebener Stroemung, Luftfahrtforschung 17, 379-386 (June, 1940). 


$$
\begin{aligned}
& \left.+\left[\gamma+\ln \left(\frac{1}{2} k\right)-F(M)\right](1-\cos \varphi)\right\} z^{\prime}(-\cos \varphi)+O\left(k^{2}\right) \\
R l\left(c_{m}\right)= & \frac{1}{2}\left(1-M^{2}\right)^{-1 / 2} \int_{0}^{\pi} d \varphi(\cos \varphi-\cos 2 \varphi) z^{\prime}(-\cos \varphi)+O(k) \\
\operatorname{Im}\left(c_{m}\right)= & \frac{1}{2} k\left(1-M^{2}\right)^{-1 / 2} \int_{0}^{\pi} d \varphi(\cos \varphi-\cos 2 \varphi) z(-\cos \varphi) \\
& \quad-\frac{1}{2} k\left(1-M^{2}\right)^{-3 / 2} \int_{0}^{\pi} d \varphi(1-\cos \varphi) \sin ^{2} \varphi z^{\prime}(-\cos \varphi)+O\left(k^{2}\right) \\
F(M)= & M^{2}+\ln \left[2 M^{-1}\left(1-M^{2}\right)\right]-\left(1-M^{2}\right)^{1 / 2} \ln ^{2}\left[M^{-1}+M^{-1}\left(1-M^{2}\right)^{1 / 2}\right] \\
= & \frac{1}{2} M^{2}\left[\left(1+\frac{1}{4} M^{2}\right) \ln \left(2 M^{-1}\right)+\frac{1}{2}-\left(\frac{17}{16}\right) M^{2}\right]+O\left(M^{6}\right) \\
= & \ln \left[2 e\left(1-M^{2}\right)\right]-\left(\frac{3}{2}\right)\left(1-M^{2}\right) \\
& \quad-\frac{1}{12}\left(1-M^{2}\right)^{2}+O\left[\left(1-M^{2}\right)^{3}\right]
\end{aligned}
$$

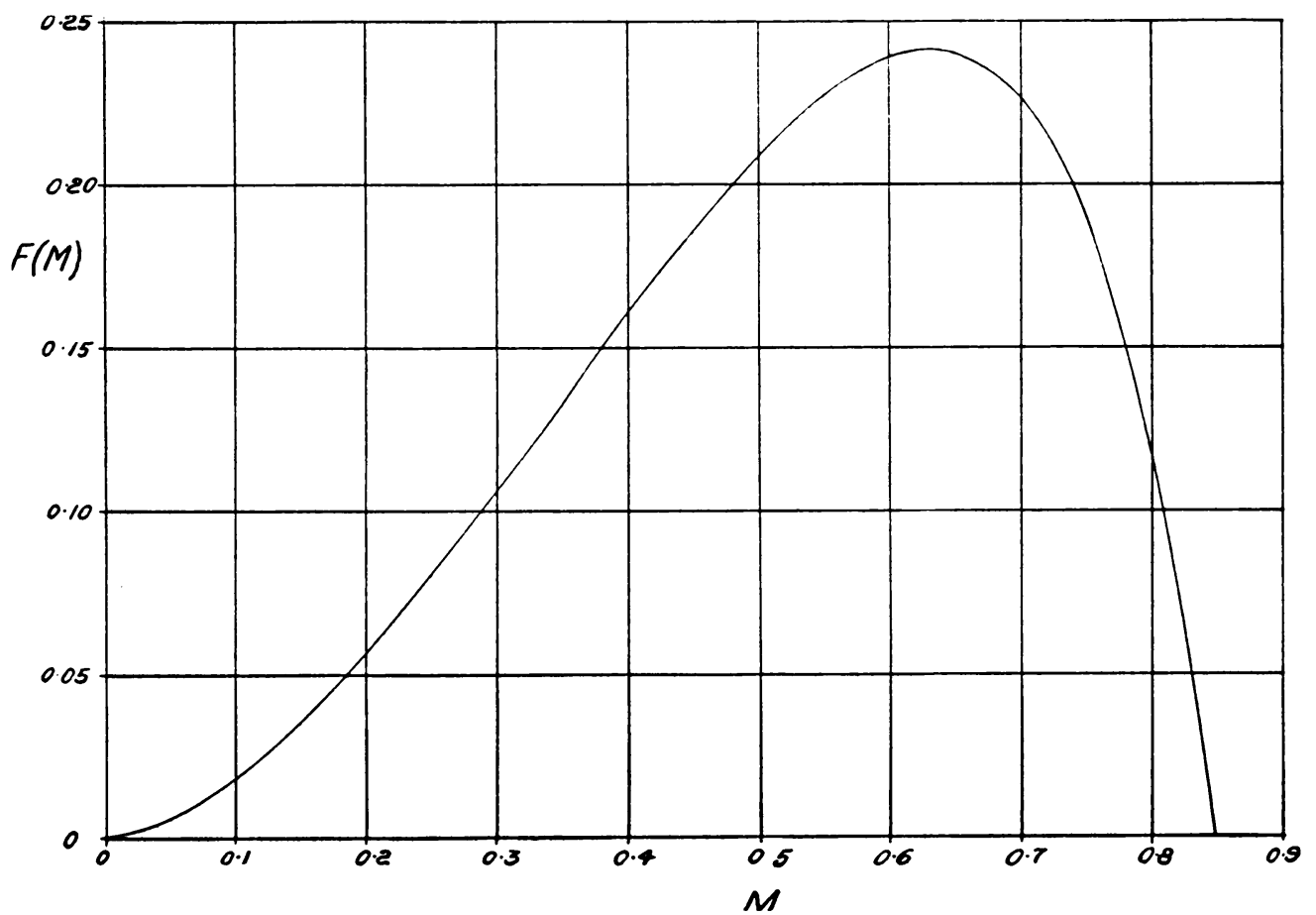

Fra. 2. $F(M)$ v8. $M$, as given by Eq. (4.7). 
where $e$ is the base of the natural logarithms. $F(M)$ is plotted in Fig. 2; for $M>0.85$ it is negative (having the value -0.26 at $M=0.9$ ), as indicated by Eq. (4.7c).

In interpreting the results (4.5) and (4.6), we observe that the real parts are simply the Munk, thin airfoil formulae multiplied by the Prandtl-Glauert correction factor for compressibility. This same statement holds for those portions of the imaginary parts associated with $z(x)$, but the contribution of $z^{\prime}(x)$ to the imaginary parts is entirely neglected by the use of the Munk formulae; moreover the compressibility correction for the latter term is considerably stronger than the Prandtl-Glauert correction.

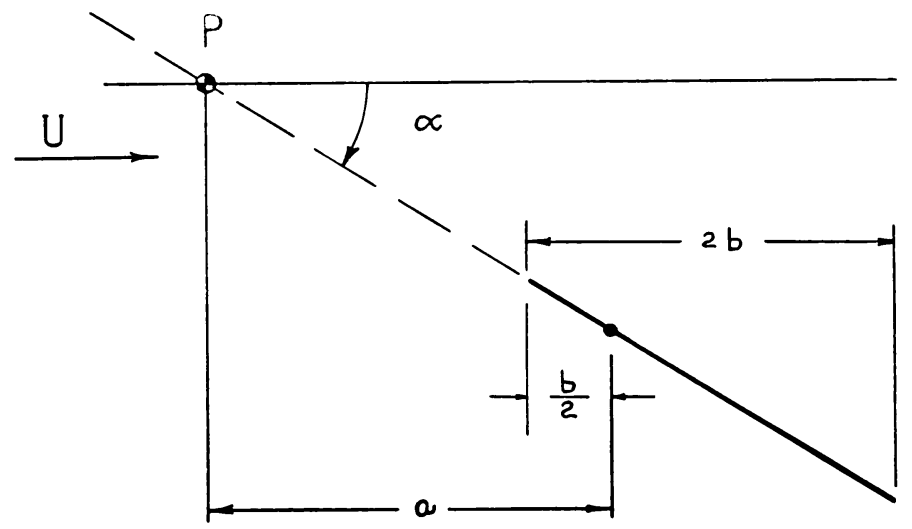

FIG. 3. Airfoil oscillating about a forward axis.

5. Pitching airfoil. As an illustrative application of the foregoing results, we consider the rotation of an airfoil about an axis $(P)$ located a distance $a$ forward of its quarterchord, as shown in Fig. 3. The displacement for unit amplitude is given by

$$
z_{\alpha}(x)=\left(\frac{1}{2}+\frac{a}{b}+x\right)
$$

Since steady-flow theory yields the real parts of the force coefficients to the desired accuracy, we consider only the imaginary parts. Eqs. (4.5b) and (4.6b) yield

$$
\begin{gathered}
\operatorname{Im}\left(c_{l \alpha}\right)=2 \pi k\left(1-M^{2}\right)^{-1 / 2}\left\{1+\left(\frac{a}{b}\right)\right. \\
\left.+\left(1-M^{2}\right)^{-1}\left[\frac{1}{2}+\gamma+\ln \left(\frac{1}{2} k\right)-F(M)\right]\right\} \\
\operatorname{Im}\left(c_{m \alpha}\right)=-\pi k\left(\frac{a}{b}\right)^{2}\left(1-M^{2}\right)^{-1 / 2}\left\{1+\left(\frac{b}{a}\right)\right. \\
+\left(\frac{b}{a}\right)\left(1-M^{2}\right)^{-1}\left[\frac{1}{2}+\gamma+\ln \left(\frac{1}{2} k\right)-F(M)\right] \\
\left.+\frac{1}{4}\left(\frac{b}{a}\right)^{2}\left(1-M^{2}\right)^{-1}\left(2-M^{2}\right)\right\}
\end{gathered}
$$


If we calculate $\operatorname{Im}\left(c_{m}\right)$ on the basis of stationary airfoil theory, the result is

$$
\operatorname{Im}\left(c_{m \alpha}\right)=-\pi k\left(\frac{a}{b}\right)^{2}\left(1-M^{2}\right)^{-1 / 2}\left[1+\frac{1}{2}\left(\frac{b}{a}\right)\right]^{2}
$$

For the tail on a typical airplane $(b / a)$ will be of the order of 0.1 , and $k$ generally will fall between 0.1 and 0.01 . Taking these values in Eqs. (5.3) and (5.4), at $M=0$ and $M=0.707$, we obtain

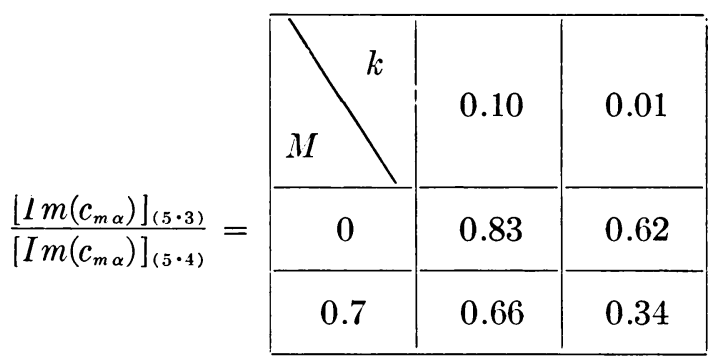

It is evident that the use of stationary airfoil theory to predict total damping in pitch (horizontal surface) or yaw (vertical surface) may be dangerously non-conservative. Moreover, we may expect similar discrepancies in the calculation of damping in pitch for a swept wing. While reliable experimental evidence is small, it tends to support this conclusion. In particular, it appears that many high speed, jet aircraft exhibit a tendency to "snake", and this may well be the result of an overly optimistic calculation of the expected damping in yaw from the vertical fin. However, no quantitative answer can be given until the questions of induction and downwash interference, (i.e. effect of wing on tail) are resolved. A brief indication as to the order of magnitude of the former effects is given in the next section. An answer to the latter question depends on the determination of the flow in the wake of the oscillating wing and is, therefore, rather more complex. An investigation of this matter is being undertaken. ${ }^{11}$

6. Three dimensional effects (Added in proof). An investigation of an elliptic wing, based on Reissner's analysis of a three dimensional oscillating wing in incompressible flow $^{12}$ and an extension of the Prandtl-Glauert rule $^{13}$, yields the result.

$$
\begin{aligned}
\operatorname{Im}\left(C_{m \alpha}\right)=-\frac{1}{2} k\left(\frac{a}{b}\right)^{2}\left(\frac{d C_{L}}{d \alpha}\right)\left\{1+2\left(\frac{b}{a}\right)\right. \\
-\left(\frac{b}{a}\right)\left(1-M^{2}\right)^{-1}(0.530+\ln A)\left[1+\left(\frac{2}{A}\right)\right]^{-1} \\
\left.-\left(\frac{b}{a}\right)^{2}\left(1-M^{2}\right)^{-1}\left[0.01+0.528\left(\frac{M^{2}}{A}\right)\right]\right\} \\
-0.820 M^{2}\left(1-M^{2}\right)^{-1}
\end{aligned}
$$

${ }^{11} \mathrm{~J}$. W. Miles, On downwash corrections in unsteady flow, J. Aero. Sci. 17, 253 (1950).

${ }^{12} \mathrm{E}$. Reissner, Effect of finite span on the airload distributions for oscillating wings, Parts I [NACA T.N. 1194 (1947)] and II [NACA T.N. 1195 (1947)].

${ }^{13} \mathrm{~J}$. W. Miles, On the compressibility correction for subsonic unsteady flow, J. Aero. Sci. 17, 181-182 (1950). 


$$
A=\left(1-M^{2}\right)^{1 / 2} \propto
$$

where the representative chord shown in Fig. 3 is taken as the mean aerodynamic chord, $\left(d C_{L} / d \alpha\right)$ is the three dimensional lift curve slope, corrected for compressibility, and $a$ is the aspect ratio.

If the results of the foregoing section are examined, it is found that the dominant term was $\ln k$, and now is effectively replaced by $-\ln A$. It appears, therefore, that the two dimensional results are directly applicable only to very large aspect ratio wings, although the effects that we have discussed may be of some considerable importance in many applications. We remark that, in view of Reissner's results for incompressible flow, we should expect the discrepancies between the two and three dimensional results to be less pronounced for larger values of the reduced frequency, so that it should not be inferred from our results that the two-dimensional work of Possio is necessarily inapplicable in typical flutter problems.

\section{RETARDED POTENTIALS OF SUPERSONIC FLOW*}

\section{BY J. C. MARTIN (National Advisory Committee for Aeronautics)}

Introduction. This paper deals with a space filled with ideal compressible fluid moving at velocities greater than sonic velocity. A formula will be derived for the velocity potential at any point in space when the conditions on the disturbing surfaces are given. The analysis is based on the linearized partial differential equation; therefore the disturbances must be small.

Use is made of the concept of the finite part of an infinite integral. This concept was first defined by Hadamard (Reference 1). It is used to overcome the difficulty that arises due to the elementary solution becoming infinite on the machcone emanating from the origin of the source. Hyperbolic vector operators and definitions. In reference (2) a vector operator was used in treating source and vortex distributions in the linearized theory of steady supersonic flow. This and other operators are defined below.

The hyperbolic gradient operator is defined as

$$
\nabla h=-\mathbf{i} \beta^{2} \frac{\partial}{\partial x}+\mathbf{j} \frac{\partial}{\partial y}+\mathbf{k} \frac{\partial}{\partial z}
$$

where $\beta$ is a constant, and $\mathbf{i}, \mathbf{j}$, and $\mathbf{k}$ are unit vectors in the $x, y, z$ directions respectively.

The divergence of the hyperbolic gradient operator is defined as

$$
\nabla^{2} h=-\beta^{2} \frac{\partial^{2}}{\partial x^{2}}+\frac{\partial^{2}}{\partial y^{2}}+\frac{\partial^{2}}{\partial z^{2}}
$$

The vector $\mathbf{n}^{1}$ is defined by the equation below

$$
\mathbf{n}^{\prime}=-\mathbf{i} \beta^{2} \nu_{1}+\mathbf{j} \nu_{2}+\mathbf{k}_{\nu_{3}}
$$

*Received November 25,1949 . This work is the basis of a masters thesis submitted to the Faculty of N. C. State College. The author is grateful to professors J. M. Clarkson, J. W. Cell, R. C. Bullock, and Jack Levine for their fruitful discussions and comments. 